\title{
Closed shells at drip-line nuclei
}

\author{
J. Dobaczewski, ${ }^{a}$ W. Nazarewicz, ${ }^{a, b, c, d}$ and T.R. Werner ${ }^{a, b, c}$ \\ ${ }^{a}$ Institute of Theoretical Physics, Warsaw University \\ ul. Hoża 69, 00-681 Warsaw, Poland \\ ${ }^{b}$ Physics Division, Oak Ridge National Laboratory \\ P.O. Box 2008, Oak Ridge, Tennessee 37831, USA \\ ${ }^{c}$ Department of Physics and Astronomy, University of Tennessee \\ Knoxville, Tennessee 37996, USA \\ ${ }^{d}$ Joint Institute for Heavy-Ion Research, Oak Ridge \\ Tennessee 37831, USA
}

\begin{abstract}
The shell structure of magic nuclei far from stability is discussed in terms of the self-consistent spherical Hartree-Fock-Bogoliubov theory. In particular, the sensitivity of the shell-gap sizes and the two-neutron separation energies to the choice of particle-hole and particle-particle components of the effective interaction is investigated.
\end{abstract}

\section{Introduction}

Shell effects in nuclei lead to characteristic irregularities in otherwise smooth behaviour of global nuclear observables when they are analyzed as functions of $Z, N$, and $A$. This applies in particular to nuclear masses, or nuclear binding energies, which conspicuously show that the closed-shell nuclei are bound stronger than open-shell systems [1-3]. Rather simple one-body potentials can account for the values of particle numbers where strong shell effects occur [magic numbers, equal to 8, 20, 28, 40, 50, 82, and (for neutrons only) 126].

Through the binding energies, many other nuclear structure properties depend on the strength of shell effects (for discussion of various binding-energy relations, see Ref. 
[4, Sec. 2). This is particularly true for the particle-emission lifetimes and/or particlecapture probabilities which dramatically depend on accessible energies. In stable nuclei these dependencies are well studied and analyzed. However, for nuclei far from stability, the relevant experimental data are scarce or simply nonexistant. For example, the nucleosynthesis of heavy elements in supernova explosions depends crucially on the values of beta decay rates and the neutron capture cross sections in very neutron-rich nuclei which do not exist in terrestrial conditions [0 [6]. A prediction of nuclear shell effects far from stability, based on the experience gained by studying stable nuclei, is an important and difficult challenge to nuclear structure theory.

In the present contribution, we aim at quantitatively describing the magnitude of shell effects in nuclei close to the neutron drip line. The description is based on the self-consistent Hartree-Fock-Bogoliubov (HFB) theory with effective interactions fitted to known nuclei. In the particle-hole (p-h) channel, we employ the Skyrme interaction. In the particle-particle (p-p, pairing) channel, several contact forces are used.

All details of the method follow Ref. [7], where the HFB theory in spatial coordinates has been introduced, and the Skyrme parametrization SkP has been obtained by a fit to properties of several magic nuclei and to the chain of the tin isotopes. In the present study, we also use the HFB method with other standard Skyrme-force parametrizations such as SIII [8] and $\mathrm{SkM}^{*}$ [9].

In drip-line nuclei, special attention should be paid to the description of pairing correlations. In particular, the condition [10, 11]:

$$
S_{n} \approx-\lambda_{n}-\Delta=0,
$$

which requires that at the drip line the neutron separation energy, $S_{n}$, vanishes, shows that pairing effects are crucial for determining the position of the one-neutron drip line. From Eq. (1) it follows that properties of the mean field, characterized by the Fermi energy, $\lambda$, (determined by the p-h component of the effective interaction) and those of the pairing field, $\Delta$, (determined by the p-p part of the effective interaction) are equally important far from the beta-stability line. Consequently, the choice of the pairing interaction is vital for analyzing shell effects in drip-line nuclei.

In the present study, results obtained with several p-h and pairing interactions (see Section 2) are confronted with the data. A comparison of theoretical predictions for the two-neutron separation energies, $S_{2 n}$, with experimental values is given in Section 3 , while in Section 4 the sizes of calculated neutron shell gaps for very neutron-rich nuclei are discussed. 


\section{Pairing interaction}

\subsection{Magnitude of pairing correlations}

Odd-even staggering of nuclear masses, i.e., the fact that nuclides with odd numbers of protons or neutrons are found to have smaller binding energy than the arithmetic mean of the binding energies of the two neighboring even-even nuclei, is a direct consequence of nuclear pairing correlations. The strength of pairing interaction is often adjusted so as to reproduce the odd-even mass difference

$$
\delta_{\mathrm{N}}=\frac{1}{2}(B(N-1, Z)+B(N+1, Z))-B(N, Z) .
$$

In particular, in Ref. [7], a certain linear combination of the parameters of the Skyrme force has been adjusted to fit the values of the odd-even mass difference in the tin isotopes. The resulting Skyrme interaction, SkP, describes both p-h and p-p channels.

In Table 1 we present the neutron odd-even mass difference for proton-magic nuclei obtained in the HFB+SkP approach. For every magic proton number, calculations were performed for one odd isotope near the bottom of the stability valley and for its two eveneven neighbours. In an odd isotope, a particle state was blocked (by the method given in Ref. [7]), and then the lowest energy was selected among different configurations. The experimental values of $\delta_{\mathrm{N}}^{\exp }$, determined from experimental binding energies of Ref. [12], are also given in Table 1. In Table 2 we present the proton odd-even mass differences with all definitions and methods mutatis mutandis analogous to those used for neutrons.

In Tables 1 and 2 we also present the values of experimental and calculated pairing gaps. The former are derived from finite-difference formulas involving experimental binding energies [13], and the latter are the average values defined according to Ref. [7]. As discussed in Ref. [1], the experimental pairing gaps are, in general, larger than the odd-even mass staggering (2).

Tables 1 and 2 show that the theoretical values of $\delta_{\mathrm{N}}$ and $\delta_{\mathrm{P}}$ are generally much smaller than the experimental values. The only exception is the odd-even mass difference for ${ }^{119} \mathrm{Sn}$ which was actually used when fitting the $\mathrm{SkP}$ force. Based on this result, one may be tempted to refit the force parameters to increase the average magnitude of pairing correlations and improve the agreement between experimental and theoretical values of $\delta$. However, a comparison of pairing-type two-body matrix elements of the SkP force with experimentally-deduced pairing matrix elements [14] indicates that they have, on the average, correct magnitude. We are therefore led to the conclusion that it is the blocking mechanism that is responsible for too low values of $\delta_{\mathrm{N}}$. 
In fact, the strength of pairing interaction in nuclei is never far away from the critical value at which, in the mean field approximation, the static pairing correlations vanish [15]. This situation precludes the use of the blocking approximation, because the critical value of pairing strength is rather large in many-quasiparticle configurations. For odd nuclei, particle number projection should, in principle, be used to describe pairing correlations near the phase transition region [16].

On the other hand, a comparison of experimental and theoretical (average) pairing gaps is less conclusive than a direct comparison of nuclear masses entering the oddeven staggering (2). This is so, because the staggering should specifically depend on the last occupied orbit near the Fermi energy, while the average pairing gap is a global property depending on all single-particle states. Until a better theory of pairing correlations in odd nuclei is applied, we do not have the correct tools which would allow the incorporation of experimental information on the odd-even staggering or on the experimental pairing gaps in a detailed adjustment of forces in the $\mathrm{p}$-p channel. Therefore, in the present study, we consider even-mass nuclei only, and consequently, we use average pairing gaps, $\tilde{\Delta}_{\mathrm{N}}$ and $\tilde{\Delta}_{\mathrm{P}}$, as qualitative measures of the pairing correlations.

The last columns of Tables 1 and 2 display proton and neutron average pairing gaps at proton and neutron magic numbers, respectively. Neither $N=40$ nor $Z=40$ shell gaps in this calculations are large enough to destroy pairing correlations. Static pairing is also obtained for the $N=20$ and 28 systems. This suggests that the magnitude of pairing correlations predicted by $\mathrm{SkP}$ is slightly too large in light nuclei. However, this is a rather subtle discrepancy, because at particle numbers 20 and 28 (both for protons and neutrons) the total energies of paired and unpaired solutions are very close. For the protons, the unpaired solution is slightly lower in energy while for the neutrons, the paired solution is favoured.

\section{$2.2 \quad$ Pairing interactions}

In the present paper we use three kinds of pairing forces. Firstly, we present the HFB results with the Skyrme force SkP. As discussed in Refs. [7,17-19] and in the previous Section, the Skyrme parametrization can be adjusted in such a way that for states near the Fermi energy it has attractive pairing matrix elements. The SkP interaction fulfills this requirement. Secondly, we employ a simple contact interaction [20-22]:

$$
V^{\delta}\left(\boldsymbol{r}, \boldsymbol{r}^{\prime}\right)=V_{0} \delta\left(\boldsymbol{r}-\boldsymbol{r}^{\prime}\right)
$$


and, thirdly, the contact interaction depending on the total (isoscalar) density of particles $\rho(\boldsymbol{r})[23-26]$

$$
V^{\delta \rho}\left(\boldsymbol{r}, \boldsymbol{r}^{\prime}\right)=\left(V_{0}+V_{3} \rho^{\gamma}\right) \delta\left(\boldsymbol{r}-\boldsymbol{r}^{\prime}\right)
$$

Since the Skyrme force is also a contact interaction, the use of the SkP parametrization in the p-p channel amounts to adding momentum-dependent and spin-exchange terms to simple forces given in Eqs. (3) and (4).

The form of the density dependence used in Eq. (4) is completely analogous to the standard Skyrme force, and hence we use an analogous notation. Parameters $V_{0}, V_{3}$, and $\gamma$ should be chosen in such a way that the interaction is strongly attractive outside the nucleus (i.e., $V_{0}$ should be large and negative) and weak inside. In this way, the pair density becomes concentrated at the surface region while for the pure contact force, Eq. (3), it is spread throughout the nuclear interior [24-28].

Parameters of phenomenological pairing interactions depend on the features of the mean field in the p-h channel, and especially on the density of single-particle states near the Fermi level. Therefore, for every parametrization of the Skyrme force used in the $\mathrm{p}$-h channel, one should use different values of parameters defining pairing interaction. In the present work, we have adjusted the strength, $V_{0}$, of the contact force (3) in the following way: For every version of the Skyrme force used in the p-h channel, spherical HFB calculations (without blocking) were performed for ${ }^{119} \mathrm{Sn}$ (see Section 2.1). Then the values of $V_{0}$ were fixed by requiring that in each case the average pairing gap, $\tilde{\Delta}_{\mathrm{N}}$, was equal to $1.256 \mathrm{MeV}$ which is the value obtained in the HFB+SkP calculations (see Table (1). When the contact interaction (3) is used in the p-p channel and the SIII, $\mathrm{SkM}^{*}$, and $\mathrm{SkP}$ parametrizations are used in the $\mathrm{p}-\mathrm{h}$ channel, we denote the resulting combined forces by $\mathrm{SIII}^{\delta}, \mathrm{SkM}^{\delta}$, and $\mathrm{SkP}^{\delta}$, respectively.

Since the density dependence in Eq. (四) introduces two additional parameters, for the purpose of the present study, we have decided to use the same values of $V_{0}$ and $\gamma$ as those used in the p-h channel, and then to fit $V_{3}$ so as to reproduce the value of $\tilde{\Delta}_{\mathrm{N}}$ in ${ }^{119} \mathrm{Sn}$. These forces are denoted by $\mathrm{SIII}^{\delta \rho}, \mathrm{SkM}^{\delta \rho}$, and $\mathrm{SkP}^{\delta \rho}$, respectively. Parameters of pairing forces used in the present study are listed in Table 3. In all cases, the phase space used, when solving the HFB equations, was chosen according to the prescription given in Ref. [7].

Average pairing gaps for semi-magic nuclei listed in Tables 1 and 2 are plotted in Figs. 1 and 2 for neutrons and protons, respectively. Open and full symbols refer to pairing forces (3) and (4), respectively. The results obtained with the SkP force agree quite satisfactorily with experimental values of odd-even staggering, apart from the neutrons in ${ }_{31}^{59} \mathrm{Ni}_{28}$ where all the forces considered fail. Neutron gaps are, in general, 
reproduced better than the proton gaps which for some forces are often much too small. Therefore, for protons one sometimes has to use a larger interaction strength $V_{0}$ than for neutrons [22, 25]. Here we are mostly interested in neutron properties and therefore we use a common value for both types of particles. Moreover, the SkP parametrization does not make such a distinction and, nevertheless, it provides a correct description of neutron and proton pairing properties simultaneously.

Density-dependent pairing interactions work better in light nuclei. On the other hand, the pure contact force seems to work better for heavier systems. No general pattern appears as far as differences between various forces used in the p-h channel are concerned. Most likely, these differences are related to details of the placement of single-particle levels near the Fermi energy which significantly vary from force to force.

As expressed by Eq. (1), to obtain a reasonable description of drip-line nuclei, pairing correlations have to be considered. However, compared with many detailed studies of Skyrme forces in the p-h channel, much less effort has been devoted to adjusting properties of pairing interactions. In stable nuclei, pairing correlations can be incorporated to some extent by using the BCS approximation, but when departing from the beta-stability valley, use of the HFB method is a must [7, 29]. A simple one-parameter fit, carried out in the present study, makes it possible to reproduce an overall magnitude of pairing correlations in the few cases discussed. The forces listed in Table 3 should be viewed as zero-order approximations, enabling us to perform the HFB analysis with standard Skyrme interactions. Certainly, more extensive fits of contact forces (possibly with additional terms included) are necessary to obtain better overall agreement with experiment. In this respect, the relative success of the pairing $\mathrm{SkP}$ force may serve as an indication that such an improvement is indeed possible.

\section{Two-neutron separation energies}

Experimental two-neutron separation energies [12], $S_{2 n}=B(N, Z)-B(N-2, Z)$, are plotted in Fig. 3(a) for five chains of proton-magic isotopes. Values quoted in Ref. [12] as "from systematics" are denoted by open symbols. Below the $S_{2 n} \simeq 10 \mathrm{MeV}$ line, experimental data are not available, and dramatic extrapolations in $N$ are required to reach the region of the r-process path $\left(S_{2 n} \approx 2 \mathrm{MeV}\right)$ or the two-neutron drip line $\left(S_{2 n}=0\right)$.

Among proton-magic, medium-heavy and heavy nuclei, there exists information about six closed neutron shells. Namely, the experimental data are available for two calcium isotopes at $N=20$ and $N=28$, two nickel isotopes at $N=28$ and $N=40$, and the zirconium and lead isotopes with $N=50$ and $N=126$, respectively. In all these cases, 
the values of $S_{2 n}$ exhibit sudden jumps of varying magnitude [12]. The size of the shell gap can be directly related to a difference between two-neutron separation energies,

$$
S_{2 n}^{N}-S_{2 n}^{N+2}=-B(N-2, Z)+2 B(N, Z)-B(N+2, Z)
$$

i.e., is related to the second derivative of the binding energy. Hence, in order to establish the size of the neutron shell gap, the mass of the doubly magic nucleus and the masses of its two neighbouring even isotopes have to be known. Similar information can be obtained by analyzing quantities $\Delta_{2 n}$ introduced in [4. However, they involve masses of four isotopes and, therefore, they are less convenient. It should be stressed that in our study we do not aim at subtracting the smooth background from the experimental masses, but rather use indicators (5) to compare theoretical and experimental masses.

A very long chain of known tin isotopes starts and terminates around neutron magic numbers $N=50$ and $N=82$. Unfortunately, so far, the masses of ${ }^{100} \mathrm{Sn}$ and ${ }^{134} \mathrm{Sn}$ have not been measured, and ${ }^{98} \mathrm{Sn}$ is probably proton-unstable. Therefore, for the purpose of the present study, we establish the size of the $N=82$ shell gap at $Z=50$ by taking the mass of ${ }^{134} \mathrm{Sn}$ from the systematic trend proposed in Ref. [12].

The neutron subshell effect at $N=40$ is rather weak, and the experimental $S_{2 n}$-values for the nickel isotopes exhibit only a rather small discontinuity [see Fig. 3(a)]. Therefore, in the following, we concentrate on neutron shell gaps obtained from the nuclear masses around ${ }_{20}^{40} \mathrm{Ca},{ }_{28}^{48} \mathrm{Ca},{ }_{28}^{56} \mathrm{Ni},{ }_{50}^{90} \mathrm{Zr},{ }_{82}^{132} \mathrm{Sn}$, and ${ }_{126}^{208} \mathrm{~Pb}$. The direct information on the isotopic dependence of the shell-gap sizes is even more restrained. Only a comparison between the shell-gap values for ${ }^{56} \mathrm{Ni}$ and ${ }^{48} \mathrm{Ca}$ suggests that the size of the $N=28$ shell gap decreases with increasing neutron excess.

In Fig. 3(b), we compare, with experimental data, the results of global mass calculations obtained in the finite-range droplet model (FRDM) of Ref. [30]. This is a microscopic-macroscopic model which is known to give a very good fit to experimental nuclear masses. (It is worth noting that the FRDM explicitly considers deformation effects.) Consequently, the two-neutron separation energies are also well reproduced in this model. Only in some specific regions of the periodic chart are some discrepancies present. For example, a sudden decrease in $S_{2 n}$ around ${ }^{96} \mathrm{Zr}$ is not reproduced and also the trend predicted for light zirconium isotopes is slightly off. As far as the jumps of $S_{2 n}$ at magic numbers, the FRDM reproduces the six data points fairly well. In particular, the best agreement is obtained for ${ }^{90} \mathrm{Zr}$; the largest discrepancy is seen in ${ }^{48} \mathrm{Ca}$.

At this point, one should note that any kind of correlation, absent in a magic nucleus but appearing in its two even neighbors, would lead to a decreased size of the shell gap (5). Indeed, in such a situation, the binding energy of the magic nucleus is expected 
to be well described by an uncorrelated state, while the correlations in the neighboring nuclei would lead to a reduced value of $S_{2 n}^{N}-S_{2 n}^{N+2}$. Consequently, if correlations (e.g., fluctuations due to pairing vibrations, shape oscillations, etc.) are missing in the model description, the differences $S_{2 n}^{N}-S_{2 n}^{N+2}$ can easily be overestimated. Of course, this kind of reasoning tacitly assumes that the effects on shell-gap sizes originating from the pure mean field are correctly described which certainly need not to be valid.

Predictions of the FRDM concerning the isotopic dependence of shell-gap sizes are very clear. With increasing neutron excess, the gap at $N=28$ decreases (too much as compared with experiment), while the gaps at $N=50$ and 82 stay practically the same. In practical terms, in the microscopic-macroscopic methods the shell-gap sizes are the direct consequences of simple parametrizations of the isotopic dependence of one-body average potential. Such dependences probably cannot be inferred, with sufficiently high precision, from fits to known nuclei, because these nuclei form isotopic chains which are not long enough. From this point of view, far-reaching extrapolations, based on twobody density-dependent effective interactions, have a better chance of success. Moreover, the results of microscopic-macroscopic methods are plagued with numerous uncertainties when they are applied to nuclei far from stability [31].

In Fig. 嗮(c), we present results obtained by the spherical HFB+SkP calculations. Here, the overall agreement with data is similar to that obtained in the FRDM. Apart from slightly too high values of $S_{2 p}$ obtained before the $N=126$ shell closure in the lead isotopes, and small deviations for some zirconium isotopes (similar as in the FRDM), the isotopic dependence of the two-neutron separation energies is reproduced fairly well. The predicted size of the magic gap is slightly too large in ${ }^{208} \mathrm{~Pb}$, slightly too small in ${ }^{90} \mathrm{Zr}$ and ${ }^{132} \mathrm{Sn}$, and seriously underestimated in the light magic nuclei. Predictions concerning the isotopic dependence of the shell-gap sizes are markedly different than those of the FRDM. With increasing neutron excess, the shell gaps at $N=50$ and 82 decrease substantially. This effect has already been observed in Refs. [11, 32] and is discussed in Section t.

After adjusting the strength of the contact force (3) to the odd-even mass staggering (Section 2.2), we are now able to perform the HFB calculations with other standard Skyrme parametrizations. The results for the $\mathrm{SIII}^{\delta}$ and $\mathrm{SkM}^{\delta}$ forces are presented in Figs. 因(a) and $⿴(\mathrm{~b})$, respectively. They are compared with the results of the $\mathrm{SkP}^{\delta}$ force

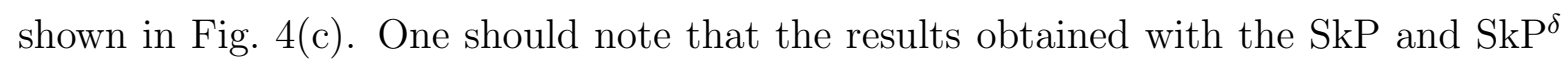
forces are very similar, in spite of their very different properties in the pairing channel.

As seen in Fig. 团, neither for $\mathrm{SIII}^{\delta}$ nor for $\mathrm{SkM}^{\delta}$ is the agreement with data satisfactory. In particular, the shell-gap sizes at $N=50,82$, and 126 are strongly overestimated, 
and the values and the slopes of $S_{2 n}$ are, in most cases, incorrect. At this point, one should note that a systematic few-MeV discrepancy in two-neutron separation energies leads to an integrated many-MeV discrepancy in total binding energies, cf. results for lead isotopes presented in Ref. [25].

The results shown in Fig. 团illustrate a very strong dependence of the two-neutron separation energies on the force parameters. Although older forces, such as SIII and $\mathrm{SkM}^{*}$, can perform well in certain regions of $Z$ and $N$, they do not give a satisfactory global reproduction of the data. On the other hand, a fairly good global agreement obtained with the $\mathrm{SkP}$ suggests that the improvement is possible, while a still better parametrization would be welcome (see Refs. [33, 34]). Of course, forces which fail in reproducing the behavior with $(N-Z)$ in known nuclei have little chance to perform better when going far from stability. For example, the almost constant values of $S_{2 n}$ obtained in the very heavy tin and lead isotopes with the $\mathrm{SIII}^{\delta}$ and $\mathrm{SkM}^{\delta}$ interactions, followed by strong shell effects at $N=126$ and $N=184$, do not seem very reliable. A detailed analysis of the force-dependence of results may give us valuable information on the relative importance of various force parameters. In the next Section, such an analysis is performed for the sizes of magic gaps in known and exotic nuclei.

\section{Magic shell gaps far from stability}

In the present Section, the $Z$-dependence of sizes of neutron shell gaps is analyzed. The shell-gap sizes are defined as in Eq. (5); six neutron magic numbers from $N=20$ to 126 are considered. In the $Z$-representation, neutron-rich nuclei appear at small proton numbers and the proton drip line is approached at large proton numbers.

\subsection{The role of deformation}

Experimental values of $S_{2 n}^{N}-S_{2 n}^{N+2}$ as functions of $Z$ are shown in Fig. 5(a). A conspicuous feature present in the data is the appearance of well-defined maxima at magic numbers. This fact can be associated with the effect of deformation. Namely, the shell-gap sizes defined in Eq. (5) depend on the masses of even isotopes adjacent to a neutron-magic system. In nuclei which are not proton-magic, both neutron and proton shells are open. In such cases, deformation effects have to be considered. Therefore, the isotopic dependencies seen in Fig. 5(a) illustrate not only the "pure" isotopic dependence of the shell effects but also the magnitude of deformation-like correlations. Of course, experimentally, one cannot easily disentangle the deformation and isotopic effects, and the complete theoretical description should take them into account simultaneously. On 
the other hand, extrapolations to nuclei far from stability are difficult enough, and one would wish to consider one problem at a time. Since, in the present study, we consider spherical shapes only, we must disregard the isotopic dependencies present in the data. Consequently, we concentrate mainly on the six cases of doubly magic nuclei discussed in Section 3. In Figs. 5(b) and 5(c), and also in the figures discussed below, these six values are denoted by full circles and, in addition, two points corresponding to the $N=28$ systems are connected by a thick line.

In spite of the fact that the FRDM results account for the deformation effects, this model does not reproduce the experimental differences $S_{2 n}^{N}-S_{2 n}^{N+2}$ in an entirely satisfactory way. The maxima at $N=20$ and $N=Z=28$ are not high enough and the maximum at ${ }^{48} \mathrm{Ca}$ is absent. The isotopic trend predicted for the light $N=50$ isotones is correct, but the proton-number dependence in the heavy $N=50$ isotones, and in the $N=82$ and 126 isotones, is visibly too weak. One should, of course, bear in mind that we are now looking at very fine details of mass predictions.

The HFB+SkP results presented in Fig. 5 (c) show very clearly that sizes of spherical shell gaps strongly depend on the neutron excess. For all neutron magic numbers, the gap sizes decrease when approaching the neutron drip line; i.e., when the proton number decreases for a fixed value of $N$. (Again, since these results are restricted to spherical shapes, one cannot expect to reproduce the experimental proton-number dependence seen in Fig. 5(a).) In the automated calculations we have performed for long chains of isotopes or isotones, in some cases, a poor convergence was obtained, due to crossings between single-particle or quasiparticle levels. Since, in this work, we discuss only general trends, we do not try to cure these cases. (This would require the full configuration blocking.) In Figs. $5-8$ the converged points are connected by straight lines.

\subsection{Hartree-Fock results}

We are now in a position to discuss possible origins of the isospin dependence of the shell-gap sizes. First of all, by using the Hartree-Fock (HF) method in which the pairing correlations are neglected, we may study the role of the interactions in the p-h channel alone. In Fig. 6 we present the HF results for three Skyrme forces, namely, SIII, SkM*, and SkP.

Let us first discuss the force-independent, generic features of the predicted shell-gap sizes. For all the forces considered, the sizes of the $N=20$ to $N=82$ shell gaps decrease with decreasing proton number. The effect is strongest for $N=28$, and then weakens for $N=50$ and $N=82$. For $N=126$, the gap size is predicted to be almost constant as a 
function of $Z$, and even a reversed trend is obtained in the HF+SIII model. In all cases, a decrease of the $N=28$ shell gap between ${ }^{56} \mathrm{Ni}$ and ${ }^{48} \mathrm{Ca}$ is predicted.

Systematically, the magnitude of the shell effect is too strong for the SIII and SkM* interactions. The sizes of the $N=50$ and $N=82$ shell gaps are overestimated by as much as a factor of two. A part of this discrepancy can be attributed to missing correlations. As discussed above, they always decrease the shell-gap sizes; the influence of pairing correlations is discussed in Section 4.3. In the HF+SkP model, the size of the $N=28$ shell gap is too small. One may therefore anticipate that it will be much too small after the pairing correlations are taken into account.

Overall magnitudes of shell-gap sizes can be clearly correlated with different values of the effective mass corresponding to the three studied Skyrme forces. Indeed, the single-particle level density $g$ at the Fermi level can be approximated by [35, 36]

$$
g=\frac{3}{4} N_{t} \frac{2 m^{*}}{\left(\hbar k_{F}\right)^{2}},
$$

where $N_{t}$ is the particle number, $k_{F}$ is the Fermi momentum, and $m^{*}$ is the effective mass. For the SIII, SkM*, and SkP, the effective masses are $m^{*} / m=0.76,0.79$, and 1.0, respectively. Therefore, the density of single-particle states increases when going from SIII to SkP, and hence the sizes of shell gaps decrease accordingly.

Interesting conclusions can be drawn when comparing sizes of the $N=20$ and $N=28$ shell gaps. In experiment, the $N=20$ gap is clearly larger $\left(\right.$ in ${ }^{40} \mathrm{Ca}$ ) than the $N=28$ gap (in ${ }^{48} \mathrm{Ca}$ ), which is the feature reproduced by the $\mathrm{HF}+\mathrm{SkP}$ model. This means that the neutron $\mathrm{f}_{7 / 2}$ orbital is closer to the upper major shell than to the lower one. On the other hand, the SIII and SkM* forces give similar sizes for both shell gaps, which may suggest that these forces place this orbital too low and have therefore too large a strength of the spin-orbit interaction (cf. discussion in Ref. [34).

\subsection{Hartree-Fock-Bogoliubov results}

By comparing results presented in Figs. 5(c) and 6(c), one may see that the pairing correlations dramatically influence the shell-gap sizes. In light nuclei, the gaps are reduced by about a factor of two, which gives substantial disagreement with experiment. One may attribute this effect to two factors: Firstly, the sizes of the $N=20$ and $N=28$ gaps obtained in the HF method are too small. This may suggest that in light nuclei the effective interaction should have an effective mass smaller than unity; this is the case for the SIII and $\mathrm{SkM}^{*}$ forces. Since both predicted shell gaps at $N=20$ and $N=28$ seem to be too small at the same time, the discrepancy with experiment should not 
be attributed to an incorrect placement of the $f_{7 / 2}$ shell, i.e., to an incorrect spin-orbit strength. Secondly, the pairing matrix elements of the SkP force are probably too strong in light nuclei. This has already been discussed in Section 2.1 in connection with the nonvanishing pairing correlations in the neutron-magic nuclei. Of course, the predicted strong pairing correlations can also result from reduced shell gaps, and both explanations strongly depend on one another.

In heavier nuclei, pairing correlations bring the shell-gap sizes, predicted by the $\mathrm{HF}+\mathrm{SkP}$ model, very close to experimental data. They also induce a dramatic quenching of shell effects in neutron-rich nuclei. For example, in the HF+SkP model, the $N=82$ shell gap decreases from about $9 \mathrm{MeV}$ to $5 \mathrm{MeV}$, with $Z$ going down from 86 to 40 . In the $\mathrm{HFB}+\mathrm{SkP}$ method, the $N=82$ gap decreases from $7 \mathrm{MeV}$ to almost zero. As a result, there is almost no influence of the $N=82$ gap on the position of the spherical neutron drip line [11].

Figures 0 and 8 display the HFB results for the shell-gap sizes obtained with the density-independent (3) and density-dependent (国) contact forces, respectively. The results are presented for the three Skyrme interactions discussed above. It can be seen that for the interactions SIII and SkM* which have low effective mass, pairing correlations are not sufficiently strong to bring the shell gaps in heavy nuclei down to the experimental values. In light nuclei, the sizes of the $N=20$ and $N=28$ shell gaps are again almost equal, and therefore either the former $\left(\right.$ for $\mathrm{SIII}^{\delta}$ ) or the latter (for $\mathrm{SkM}^{\delta}$ ) values agree with experiment, but a consistent description cannot be obtained.

In general, the shell-gap sizes obtained with the density-dependent pairing interaction are lower than those obtained with the pure contact force. The differences between different p-p interactions are significant. For example, the $N=82$ shell gaps predicted in the $\mathrm{SkM}^{\delta}$ calculations are always greater than $5 \mathrm{MeV}$, while those obtained in the $\mathrm{SkM}^{\delta \rho}$ version exhibit a pronounced quenching when approaching the neutron drip line.

\section{Conclusions}

The results of self-consistent HF and HFB calculations with several Skyrme-type interactions demonstrate that the magnitude of shell effects at magic numbers strongly depends on force parameters. In the p-h channel, the main factor is the effective mass which determines the single-particle level density around the Fermi level. In the pairing channel, the density-dependence of a p-p interaction plays a very important role. Both effects combined, i.e., the increase in $\mathrm{m}^{*} / \mathrm{m}$ and the presence of density-dependent pairing, lead to the shell-quenching effects in nuclei far from the beta-stability line. 
This result seems to be consistent with the recent analysis of solar-system r-process abundance distributions [37].

Our brief analysis indicates that a lot of systematic work still needs to be done to obtain a "universal" Skyrme-type interaction. For instance, the density dependence of the effective interaction invites a much deeper analysis. (In principle, the force should depend on both the isoscalar density, $\rho_{n}+\rho_{p}$, and the isovector density, $\rho_{n}-\rho_{p}$ [38, 39]; the latter might be important in the description of exotic nuclei with a significant neutron excess.) Also, the interplay between deformation and pairing in open-shell drip-line nuclei is still awaiting explanation.

\section{Acknowledgements}

This research was supported in part by the Polish State Committee for Scientific Research under Contract No. 2045091 01. Oak Ridge National Laboratory is managed for the U.S. Department of Energy by Martin Marietta Energy Systems, Inc. under Contract No. DE-AC05-84OR21400. The Joint Institute for Heavy Ion Research has as member institutions the University of Tennessee, Vanderbilt University, and the Oak Ridge National Laboratory; it is supported by the members and by the Department of Energy through Contract No. DE-FG05-87ER40361 with the University of Tennessee. Theoretical nuclear physics research at the University of Tennessee is supported by the U.S. Department of Energy through Contract No. DE-FG05-93ER40770.

\section{References}

[1] A. Bohr and B.R. Mottelson, Nuclear Structure, vol. 1 (W.A. Benjamin, New York, 1969).

[2] W.D. Myers and W.J. Swiatecki, Ann. Rev. Nucl. Part. Sci. 32, 309 (1982).

[3] I. Ragnarsson and R.K. Sheline, Phys. Scr. 29, 385 (1984).

[4] A.S. Jensen, P.G. Hansen and B. Jonson, Nucl. Phys. A431, 393 (1984).

[5] W.M. Howard, S. Goriely, M. Rayet, and M. Arnould, Astrophys. J. 417, 713 (1993).

[6] K.-L. Kratz, J.-P. Bitouzet, F.-K. Thielemann, P. Möller, and B. Pfeiffer, Astrophys. J. 403, 216 (1993). 
[7] J. Dobaczewski, H. Flocard and J. Treiner, Nucl. Phys. A422, 103 (1984).

[8] M. Beiner, H. Flocard, N. Van Giai and P. Quentin, Nucl. Phys. A238, 29 (1975).

[9] J. Bartel, P. Quentin, M. Brack, C. Guet, and H.B. Håkansson, Nucl. Phys. A386, 79 (1982).

[10] M. Beiner, R.J. Lombard, and D. Mas, Nucl. Phys. A249, 1 (1975).

[11] R. Smolańczuk and J. Dobaczewski, Phys. Rev. C48, R2166 (1993).

[12] G. Audi and A.H. Wapstra, Nucl. Phys. A565, 1 (1993); Nucl. Phys. A565, 66 (1993).

[13] P. Möller and J.R. Nix, Nucl. Phys. A536, 20 (1992).

[14] J.P. Schiffer and W.W. True, Rev. Mod. Phys. 48, 191 (1976).

[15] P. Ring and P. Schuck, The Nuclear Many-Body Problem (Springer-Verlag, 1980).

[16] H.J. Mang, J.K. Poggenburg, and J.O. Rasmussen, Nucl. Phys. 64, 353 (1965).

[17] M. Waroquier, J. Sau, K. Heyde, P. Van Isacker, and H. Vincx, Phys. Rev. C19, 1983 (1979).

[18] Nguyen Van Giai and H. Sagawa, Phys. Lett. B106, 379 (1981).

[19] M. Waroquier, K. Heyde, and G. Wenes, Nucl. Phys. A404, 269 (1983); A404, 298 (1983).

[20] F. Tondeur, Nucl. Phys. A315, 353 (1979).

[21] M.V. Zverev and E.E. Sapershtein, Sov. J. Nucl. Phys. 42, 683 (1985).

[22] S.J. Krieger, P. Bonche, H. Flocard, P. Quentin, and M.S. Weiss, Nucl. Phys. A517, 275 (1990).

[23] R.R. Chasman, Phys. Rev. C14, 1935 (1976).

[24] V.E. Starodubsky and M.V. Zverev, Phys. Lett. B 276, 269 (1992).

[25] N. Tajima, P. Bonche, H. Flocard, P.-H. Heenen, and M.S. Weiss, Nucl. Phys. A551, 434 (1993). 
[26] S.A. Fayans, S.V. Tolokonnikov, E.L. Trykov, and D. Zawischa, Phys. Lett. B (1994).

[27] S.T. Belyaev, A.V. Smirnov, S.V. Tolokonnikov, and S.A. Fayans, Sov. J. Nucl. Phys. 45, 783 (1987).

[28] J. Dobaczewski, W. Nazarewicz, and T.R. Werner, to be published.

[29] W. Nazarewicz, J. Dobaczewski, and T.R. Werner, Physica Scripta, in press.

[30] P. Möller, J.R. Nix, W.D. Myers, and W.J. Swiatecki, At. Data and Nucl. Data Tables, to be published.

[31] W. Nazarewicz, T.R. Werner, and J. Dobaczewski, Phys. Rev. C, in press.

[32] J. Dobaczewski, I. Hamamoto, W. Nazarewicz, and J.A. Sheikh, Phys. Rev. Lett. 72, 981 (1994).

[33] J. Meyer, private communication, 1994.

[34] P.-G. Reinhard and H. Flocard, preprint 1994.

[35] B. Grammaticos and A. Voros, Ann. Phys. (NY) 123, 359 (1979).

[36] A.S. Jensen and A. Miranda, Nucl. Phys. A449, 331 (1986).

[37] K.-L. Kratz, preprint IKMz 94-3; to appear in Proc. 3rd Int. Symp. on Nuclear Astrophysics "Nuclei in the Cosmos", 1994.

[38] J. Dąbrowski, Nukleonika 22, 143 (1977).

[39] U. Regge and D. Zawischa, Phys. Rev. Lett. 61, 149 (1988). 
Table 1: Experimental and calculated neutron odd-even mass difference $\delta_{\mathrm{N}}^{\exp }$ and $\delta_{\mathrm{N}}$ for selected proton-magic nuclei. Experimental pairing gaps $\Delta_{N}^{\exp }$ (determined as in [13]) and calculated neutron and proton average pairing gaps $\tilde{\Delta}_{\mathrm{N}}$ and $\tilde{\Delta}_{\mathrm{P}}$ are also given.

\begin{tabular}{cc|ccccc}
\hline$N$ & $Z$ & $\delta_{\mathrm{N}}^{\exp }$ & $\delta_{\mathrm{N}}$ & $\Delta_{\mathrm{N}}^{\exp }$ & $\tilde{\Delta}_{\mathrm{N}}$ & $\tilde{\Delta}_{\mathrm{P}}$ \\
\hline 23 & 20 & 1.600 & 1.301 & 1.708 & 1.584 & 0.000 \\
31 & 28 & 1.194 & 0.234 & 1.446 & 0.720 & 0.000 \\
53 & 40 & 0.743 & 0.448 & 0.829 & 0.712 & 0.793 \\
69 & 50 & 1.312 & 1.293 & 1.378 & 1.256 & 0.000 \\
121 & 82 & 0.736 & 0.634 & 0.801 & 0.737 & 0.000 \\
\hline
\end{tabular}

Table 2: Same as in Table 1, except for protons replacing neutrons and vice versa.

\begin{tabular}{cc|ccccc}
\hline$N$ & $Z$ & $\delta_{\mathrm{P}}^{\exp }$ & $\delta_{\mathrm{P}}$ & $\Delta_{\mathrm{P}}^{\exp }$ & $\tilde{\Delta}_{\mathrm{P}}$ & $\tilde{\Delta}_{\mathrm{N}}$ \\
\hline 20 & 17 & 0.929 & 0.496 & 1.535 & 1.191 & 1.202 \\
28 & 25 & 1.147 & 0.709 & 1.540 & 1.139 & 0.605 \\
40 & 31 & 0.938 & 0.303 & 1.385 & 0.900 & 1.319 \\
50 & 37 & 0.997 & 0.514 & 1.356 & 0.976 & 0.000 \\
82 & 59 & 0.998 & 0.664 & 1.229 & 0.931 & 0.000 \\
126 & 85 & 0.661 & 0.530 & 0.846 & 0.552 & 0.000 \\
\hline
\end{tabular}


Table 3: Parameters of pairing forces used in the present study. Columns denoted by $\delta$ and $\delta \rho$ give parameters of forces (3) and (41), respectively. In the latter case, parameters $V_{0}$ and $\gamma$ are identical to those in the p-h channel. Different sets of parameters are fitted to different forces used in the p-h channel, as indicated in the first column.

\begin{tabular}{c||c|ccc}
\hline \multirow{2}{*}{$\begin{array}{c}\text { Force in the } \\
\text { p-h channel }\end{array}$} & $\delta$ & \multicolumn{3}{|c}{$\delta \rho$} \\
\cline { 2 - 5 } & $V_{0}\left(\mathrm{MeV} \mathrm{fm}^{3}\right)$ & $V_{0} \equiv t_{0}\left(\mathrm{MeV} \mathrm{fm}^{3}\right)$ & $V_{3}\left(\mathrm{MeV} \mathrm{fm}^{3+\gamma}\right)$ & $\gamma \equiv \alpha$ \\
\hline SIII & -160 & -1128.75 & 50715 & 1 \\
$\mathrm{SkM}^{*}$ & -173 & -2645 & 21591 & $1 / 6$ \\
$\mathrm{SkP}$ & -185 & -2931.696 & 24175 & $1 / 6$ \\
\hline
\end{tabular}


Figure captions

Figure 1: Average neutron pairing gaps, $\tilde{\Delta}_{\mathrm{N}}$, for proton-magic nuclei listed in Table 1 . Odd-even staggering parameter $\delta_{\mathrm{N}}^{\exp }$ is plotted as the experimental value. All forces were adjusted so as to give identical results for ${ }_{69}^{119} \mathrm{Sn}_{50}$.

Figure 2: Average proton pairing gaps, $\tilde{\Delta}_{\mathrm{P}}$, for neutron-magic nuclei listed in Table 2 . Odd-even staggering parameter $\delta_{\mathrm{P}}^{\exp }$ is plotted as the experimental value.

Figure 3: Two-neutron separation energies, $S_{2 n}$, for proton-magic isotopes. In (a), experimental (full circles) and "from systematics" [12 (open circles) values are shown. In (b) and (c) compared with the experimental data are the results of the FRDM [30] and of the $\mathrm{HFB}+\mathrm{SkP}$ model, respectively.

Figure 4: Two-neutron separation energies, $S_{2 n}$, for proton-magic isotopes. The HFB results with the $\mathrm{SIII}^{\delta}$ (a), $\mathrm{SkM}^{\delta}$ (b), and $\mathrm{SkP}^{\delta}$ (c) parametrizations (see Section 2.2) are compared with the experimental data.

Figure 5: Sizes of neutron shell gaps as functions of the number of protons. Experimental data (a) are compared with the results of the FRDM [30 (b) and of the HFB+SkP method (c). Deformation effects are included in the FRDM while only spherical shapes are considered in $\mathrm{HFB}+\mathrm{SkP}$. In (b) and (c), experimental data are shown only for magic nuclides and the thick line connects experimental points for two magic $N=28$ systems.

Figure 6: Sizes of neutron shell gaps obtained within the HF approximation for three parametrizations of the Skyrme force: SIII (a), SkM* (b), and SkP (c). Experimental data are shown only for magic nuclides and the thick line connects two experimental $N=28$ points. 
Figure 7: Sizes of neutron shell gaps obtained within the HFB approximation for three parametrizations of the Skyrme force: $\mathrm{SIII}^{\delta}$ (a), $\mathrm{SkM}^{\delta}$ (b), and $\mathrm{SkP}^{\delta}$ (c). Contact pairing force (3) is used. The same experimental data as in Fig. 6 are shown.

Figure 8: Sizes of neutron shell gaps obtained within the HFB approximation for three parametrizations of the Skyrme force: $\operatorname{SIII}^{\delta \rho}$ (a), $\mathrm{SkM}^{\delta \rho}$ (b), and $\mathrm{SkP}^{\delta \rho}$ (c). Densitydependent contact pairing force (4) is used. The same experimental data as in Fig. 6 are shown. 